\title{
Cost-Utility of Quadrivalent Versus Trivalent Influenza Vaccine in Germany, Using an Individual-Based Dynamic Transmission Model
}

\author{
Christiaan Dolk $^{1}$ (D) Martin Eichner $^{2,3} \cdot$ Robert Welte $^{4} \cdot$ Anastassia Anastassopoulou $^{4} \cdot$ \\ Laure-Anne Van Bellinghen ${ }^{5}$ - Barbara Poulsen Nautrup ${ }^{6}$. Ilse Van Vlaenderen ${ }^{5}$. \\ Ruprecht Schmidt-Ott ${ }^{4,7} \cdot$ Markus Schwehm $^{8} \cdot$ Maarten Postma $^{1,9,10}$
}

Published online: 19 September 2016

(C) The Author(s) 2016. This article is published with open access at Springerlink.com

\begin{abstract}
Background Seasonal influenza infection is primarily caused by circulation of two influenza A strain subtypes and strains from two B lineages that vary each year. Trivalent influenza vaccine (TIV) contains only one of the two B-lineage strains, resulting in mismatches between vaccine strains and the predominant circulating $\mathrm{B}$ lineage. Quadrivalent influenza vaccine (QIV) includes both B-lineage strains. The objective was to estimate the costutility of introducing QIV to replace TIV in Germany.

Methods An individual-based dynamic transmission model (4Flu) using German data was used to provide realistic estimates of the impact of TIV and QIV on age-specific influenza infections. Cases were linked to health and economic outcomes to calculate the cost-utility of QIV versus TIV, from both a societal and payer perspective. Costs and
\end{abstract}

effects were discounted at 3.0 and $1.5 \%$ respectively, with 2014 as the base year. Univariate and probabilistic sensitivity analyses were conducted.

Results Using QIV instead of TIV resulted in additional quality-adjusted life-years (QALYs) and cost savings from the societal perspective (i.e. it represents the dominant strategy) and an incremental cost-utility ratio (ICUR) of $€ 14,461$ per QALY from a healthcare payer perspective. In all univariate analyses, QIV remained cost-effective (ICUR $<€ 50,000)$. In probabilistic sensitivity analyses, QIV was cost-effective in $>98$ and $>99 \%$ of the simulations from the societal and payer perspective, respectively.

Conclusion This analysis suggests that QIV in Germany would provide additional health gains while being costsaving to society or costing $€ 14,461$ per QALY gained from the healthcare payer perspective, compared with TIV.

C. Dolk and M. Eichner contributed equally to this work.

Electronic supplementary material The online version of this article (doi:10.1007/s40273-016-0443-7) contains supplementary material, which is available to authorized users.

Christiaan Dolk

christiaandolk@gmail.com

1 PharmacoEpidemiology and PharmacoEconomics, University of Groningen, Antonius Deusinglaan 1, 9713 GZ Groningen, The Netherlands

2 Epimos GmbH, Uhlandstrasse 3, 72144 Dusslingen, Germany

3 Clinical Epidemiology and Applied Biometry, Tübingen University, Silcherstrasse 5, 72076 Tübingen, Germany

4 GSK Germany, Prinzregentenplatz 9, 81675 München, Germany
5 CHESS, Zwarte Leeuwstraat 69, 2820 Bonheiden, Belgium

6 EAH-Consulting, Karlsgraben 12, 52064 Aachen, Germany

GSK Vaccines, Avenue Fleming 20, 1300 Wavre, Belgium

8 ExploSYS GmbH, Otto-Hahn-Weg 6, 70771

Leinfelden-Echterdingen, Germany

9 Institute for Science in Healthy Aging and healthcaRE (SHARE), University Medical Center Groningen (UMCG), Hanzeplein 1, Groningen 9713 GZ, The Netherlands

10 Department of Epidemiology, University Medical Center Groningen (UMCG), Hanzeplein 1, Groningen 9713 GZ, The Netherlands 


\section{Key Points for Decision Makers}

Replacing the trivalent influenza vaccine with the quadrivalent vaccine in Germany may reduce morbidity and mortality from influenza.

The quadrivalent vaccine provides more health benefits while being cost-saving, from the societal perspective.

The health gains achieved result in a cost-utility ratio of under $€ 15,000 / \mathrm{QALY}$ from the healthcare payer perspective.

\section{Introduction}

Seasonal influenza is an acute viral infection causing mild to severe illness or even death, especially in high-risk patients. The global annual attack rate is estimated at $5-10 \%$ for adults and $20-30 \%$ for children, resulting in a significant economic burden to society due to increased medical resource utilisation and loss of productivity [1]. Two types of influenza virus, $\mathrm{A}$ and $\mathrm{B}$, cause seasonal influenza, with similar symptoms [2-4]. Influenza A virus can be further subdivided into influenza A subtypes H1N1 and $\mathrm{H} 3 \mathrm{~N} 2$, which currently co-circulate with two lineages of influenza B virus, notably B/Yamagata and B/Victoria. The predominant circulating virus subtype or lineage differs each year. German influenza surveillance data from $2001 / 2002$ to $2014 / 2015$ show that type B viruses caused $29 \%$ of influenza [4].

Vaccination is currently the most effective strategy to prevent illness [1], with increased effectiveness when the antigenic composition of the vaccine matches the circulating virus types [5]. The World Health Organization (WHO) revises its recommendations annually regarding the appropriate antigenic composition of vaccines for the northern and southern hemispheres, on the basis of anticipated circulating variants of the virus. In Germany, influenza vaccination is recommended for people aged 60 years and older, as well as pregnant women and people with chronic medical conditions [6]. The trivalent influenza vaccine (TIV), which covers both A strain subtypes and one of the two B lineages, is currently the most used vaccine. However, mismatches between the $B$ lineage in the vaccine and the predominant circulating $\mathrm{B}$ virus frequently occur; in fact roughly $50 \%$ of circulating B virus did not match vaccine antigens in Germany during $2001 / 2002$ to $2014 / 2015$ seasons [4]. Therefore, in this period, the chances for a trivalent vaccine to match the circulating type B viruses were only about $50 \%$. Thus, since the 2012/2013 season, the WHO also recommended the option of using quadrivalent influenza vaccine (QIV) to provide broader protection against influenza $B$ viruses, as QIV vaccines protect against both influenza B lineages in addition to both A strain subtypes [7].

Studies have already investigated the impact of QIV and found it to be cost-effective in other countries; however, these data are still lacking for Germany [8-10]. When modelling an infectious disease, dynamic models are preferred over static models because they explicitly model the non-linear spread of infection over time within a population, using contact patterns between different age groups. Thus, they provide more accurate estimates of the impact of vaccination, including direct protective effects as well as indirect herd protection effects among unvaccinated people. Typically, a deterministic approach and compartmental design are used to model transmission dynamics [11]. The use of an individual-based modelling approach that takes a stochastic rather than deterministic approach enhances flexibility and allows for more realistic assumptions.

As such, a modified version of the previously published stochastic and individual-based simulation tool (4Flu) was used to model the effects of vaccination on transmission of the four influenza viruses [12]. This model extended the standard SEIR (susceptible-exposed-infected-recovered) model by factoring in acquisition of maternal antibody protection, and boosting and loss of immunity after infection and vaccination. The influenza incidence by age predicted from the transmission model was linked to health outcomes and costs from influenza event pathways as published by Van Bellinghen et al. 2014 and adapted to Germany, with the objective of assessing the cost-utility of introducing QIV to replace TIV in Germany [8].

\section{Methods}

\subsection{Model Overview}

The individual-based transmission model 4 Flu predicted the number of influenza infections by age, over 20 years, with the currently used TIV and alternatively with QIV replacing TIV. The calculation of (symptomatic) cases derived from the predicted incidence was based on the meta-analysis of Carrat et al. [13]. The epidemiologic outcomes of the $4 \mathrm{Flu}$ model were linked to economic parameters of resource use and costs in a new model named "e4Flu" (economic 4Flu), as reflected in Fig. 1. Cases were linked to medical resource use and work loss associated with uncomplicated influenza, influenza 


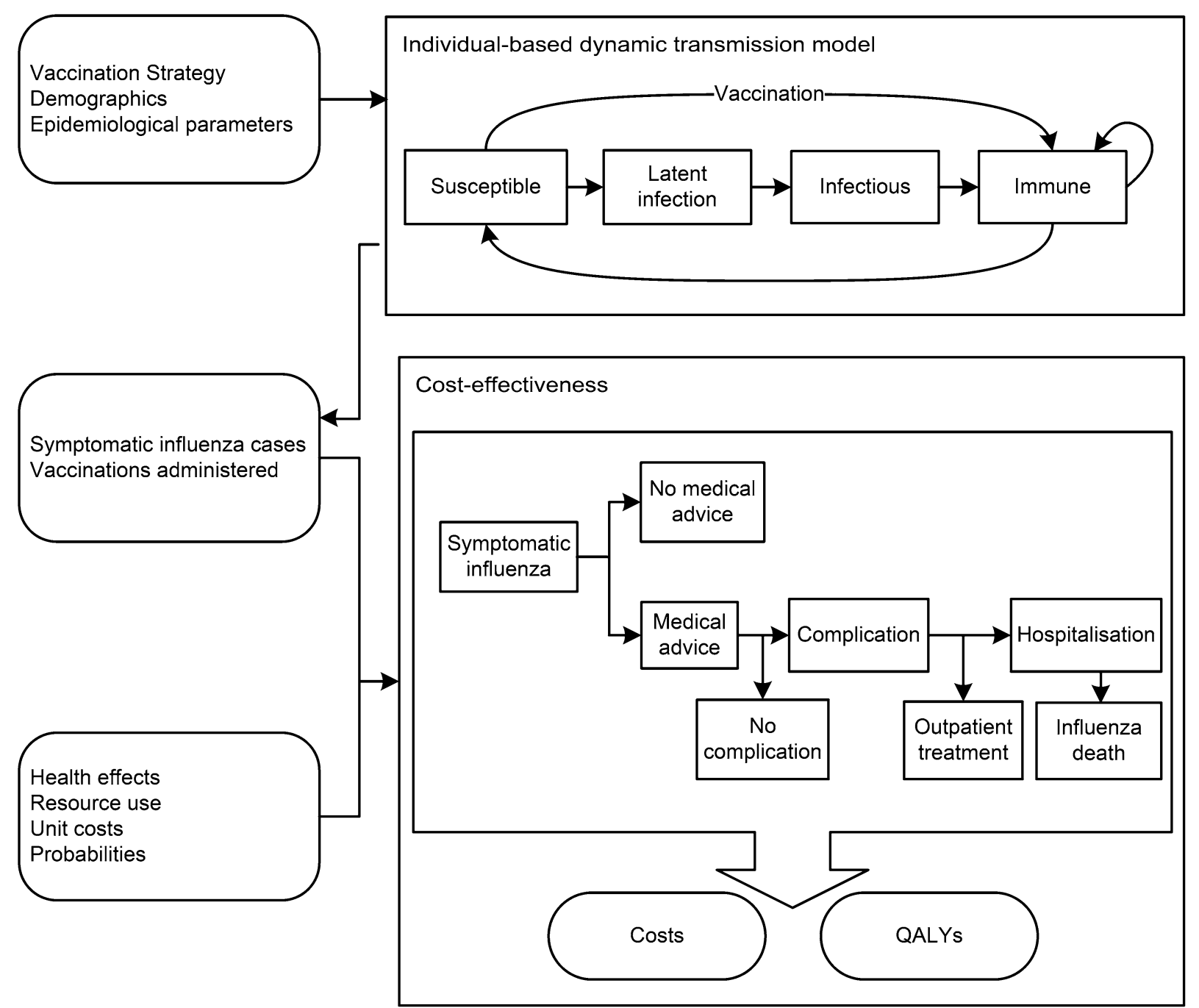

Fig. 1 Conceptual model for e4Flu depicting inputs and outputs and the epidemiologic and economic pathways. QALYs quality-adjusted lifeyears

complications and deaths. Utilities and costs were applied in order to estimate the health-related benefits [expressed in quality-adjusted life-years (QALYs)] and costs for each arm, from a payer and societal perspective. Costs were discounted at $3.0 \%$ and QALYs at $1.5 \%$ after the first year, based on recent European economic guidelines for assessing vaccines and Germany-specific discount rate assessments $[14,15]$. The modelled cohort of 100,000 people mirrored current German demographics [12]. Because of the projected declining German population size, the number of simulated individuals also decreased slightly during the simulation period. Epidemiologic and economic outcomes accumulated over a 20 -year time horizon were divided by 20 and multiplied by the ratio adjusted to the German population (81.3 million) to extrapolate annual outcomes for the entire German population [16].

\subsection{Model Inputs and Assumptions}

The spread of influenza was simulated using the stochastic individual-based simulation tool $4 \mathrm{Flu}$, which is freely available from https://www.4flu.net $[12,17]$. The simulations considered realistic annual vaccination campaigns and allowed for the independent transmission of four different influenza strains $[\mathrm{A}(\mathrm{H} 1 \mathrm{~N} 1), \mathrm{A}(\mathrm{H} 3 \mathrm{~N} 2), \mathrm{B} /$ Victoria and $\mathrm{B} /$ Yamagata] in a population whose age structure represented official German demographic statistics. Demographic changes were implemented to match the demography to that of Germany throughout the simulation period. Individuals either belonged to groups without risk or with increased risk, which influenced their disease course and likelihood of being vaccinated. Contacts between individuals, leading to transmission of any of the 
four strains independently, were based on the POLYMOD study's German contact matrix. In general, connections between individuals were kept fixed (leading to immunologic patterns in the population after influenza transmission), but sections of the contact network continuously needed to be changed, as individuals' ages increased and their average connectivity deviated from the age-dependent goals set by the POLYMOD matrix [18]. Acquired immunity was lost over time, but could be boosted by subsequent infection or vaccination. Natural infection provided an average of 9.13 years protection against similar strains, whereas vaccination protected for 1.8 years, on average [12]. The four circulating influenza strains were replaced by new drift variants of the four strains, introduced randomly in specific years in the simulation; their occurrence mimicked an additional loss of immunity and sometimes resulted in reduced vaccine efficacy. B-lineage cross protection was considered in the model. Vaccination was assumed to be performed annually in October and November. Vaccination coverage depended on the individuals' age and on whether they had a higher risk of developing health problems due to chronic disease. Individuals who were vaccinated in the previous season were preferentially vaccinated again. The current influenza vaccination policy in Germany is to vaccinate children aged 2-17 years with increased health-related risks, preferably using live attenuated influenza vaccine (LAIV) in those aged 6 years or younger. As only few children currently receive LAIV, this was not included in this model [19]. Virus transmission was subject to $43 \%$ seasonal fluctuation with a peak at the beginning of winter. The transmission model was calibrated so that $10.6 \%$ of young adults were infected in the $2006 / 07$ season, using the median of 1000 simulations [20]. Vaccine efficacy was assumed to be independent of the virus subtype or lineage and was estimated at $45,39,69,73$ and $58 \%$ for a wellmatched vaccine for age groups $0-1,2-5,6-15,16-64$ and $65+$, respectively [12]. More details of the 4 Flu model applied to the German context were published by Eichner et al. [12]. Each simulation ran for 40 years. The 20-year run-in period (starting on 1 July 1994) was primarily needed to make sure that the following evaluation period started with a realistic age-dependent immunity pattern for the population. The second 20-year period (starting on 1 July 2014) was used for the evaluation of results. During the run-in period, TIV vaccinations were performed, using the recorded B-lineage composition of the vaccine. During the 20-year evaluation period, simulations ran in parallel, using either TIV (with random choice of the B lineage each year) or QIV. The annual numbers of infections by age and risk status were translated into numbers of clinical cases [13]. The annual differences in number of clinical cases between the TIV and the QIV arms were the basis of the economic analysis. As simulations were determined by random events, the average of 1000 simulations was used for calculation of the baseline results and for each parameter variation in univariate analyses. The event pathway following symptomatic influenza, depicting resource use and health outcomes (e.g. complications, mortality), can be found in "Online resource 1" (see the electronic supplementary material). All detailed model input tables can be found in "Online resource 2".

\subsection{Resource Use, Cost and Utility Inputs and Assumptions}

Clinical influenza cases, stratified by age and risk group, followed event pathways adapted to the German context [8]. Individuals with influenza could seek medical advice (MA) from a general practitioner (GP) or visit an accident and emergency (A\&E) department. GPs could offer effective antiviral treatment with neuraminidase inhibitors (NIs). Influenza-related complications (respiratory or nonrespiratory) could develop. Respiratory complications included bronchitis, pneumonia or upper respiratory tract infection; non-respiratory complications included cardiac, renal or central nervous system complications, otitis media or gastrointestinal bleeding. Hospitalisation or outpatient treatment could follow. In the event of hospitalisation, the patient could die of the influenza-related complications. As influenza can be more severe in patients with underlying medical illness, these patients were represented in the model in an "at-risk" group (see "Online resource 2"). The model did not consider potential chronic sequelae and associated rehabilitation costs resulting from influenza infection. Adverse effects from vaccination were excluded from the model as these effects were assumed equal for QIV and TIV [21, 22]. Costs and utilities were assigned to each health state. A comprehensive literature review was performed (see "Online resource 2") to obtain model input data from published sources, government reports and systematic reviews. German utilities for influenza were not available; therefore, published data from comparable countries (the UK and Belgium) were used (see "Online resource 2"). The healthcare payer perspective also included, besides reimbursed medical costs, costs due to the German child sickness benefit ("Kinderkrankengeld") for parental absenteeism to care for a sick child up to the age of 12 years. Societal health insurance has to cover the associated net earnings in Germany. The societal perspective included, in addition to payer perspective costs, the non-reimbursable medical costs, non-medical (i.e. transportation) costs and indirect costs. Indirect costs included productivity loss due to influenza (in adults aged 18-64 years, including absenteeism of parents with a sick child) and discounted present value of lifetime future 
earnings in case of premature mortality, calculated using the human capital approach. Transportation costs of subjects (for vaccination, influenza treatment or hospitalisation) were included in the non-medical costs. All cost data were expressed at 2014 Euro price levels.

\subsection{Scenario Analyses}

The effect on outcomes of using different discount rates (i.e. 0 and $3 \%$ for both costs and effects, respectively), of increasing the QIV vaccine price, of excluding productivity losses from caregivers taking care of infants, and of replacing the human capital approach with the friction method for lost productivity calculations in premature deaths was assessed.

\subsection{Univariate and Probabilistic Sensitivity Analyses}

Key vaccine, epidemiologic and economic parameters were varied individually in one-way sensitivity analyses to assess the impact on the cost-utility ratio from the payer perspective. These parameters included vaccine efficacy, duration of immunity (natural or vaccine induced), amount of influenza B cross protection after vaccination or infection, vaccine efficacy in case of drift mismatch, percentage of symptomatic cases, probability of death after hospitalisation for respiratory complications, and disutilities associated with influenza.

To assess parameter uncertainty, a probabilistic sensitivity analysis (PSA) was performed varying both clinical and economic parameters together based on defined distributions. To evaluate the transmission parameter uncertainty within the individual-based transmission model, a second-order Monte Carlo simulation was performed [23]. Values were randomly drawn for each parameter from their associated distributions. This procedure was repeated 1000 times in the outer loop and resulted in 1000 parameter sets representing the PSA. To minimise noise as a result of stochastic uncertainty, an average outcome of 100 epidemiologic simulations was calculated for each of the 1000 parameter sets in the inner loop of the Monte Carlo simulation. PSA sampling distributions were parameterised so that $95 \%$ of samples were taken within the $95 \%$ confidence intervals of the sampled parameters; the upper and lower bounds of these confidence intervals were also used in the Tornado plot. The number of clinical influenza cases resulting from the individual-based transmission model was coupled to random values from the probabilistic distributions of economic parameters.

At this time, there is no official cost per QALY threshold in Germany. As an estimate, we applied the
$€ 50,000$ per QALY threshold that is commonly used in European and German economic evaluations [24, 25].

\section{Results}

\subsection{Base Case Results}

In an average influenza season, QIV was predicted to further reduce the burden of influenza compared with TIV in the German population, preventing an additional 276,505 cases $(4 \%)$ and 262 deaths $(6.4 \%)$ (Table 1). While the vaccination costs were higher with QIV compared with TIV, these were partially offset by cost savings due to decreased medical resource use from hospitalisation and medical visits prevented, and reduced child sickness benefits (payer perspective). From a societal perspective, vaccination with QIV instead of TIV resulted in net cost savings. Thus, QIV increased health benefits (3975 QALYs) at an additional cost ( $€ 57.5$ million) from the payer perspective and at a cost saving ( $€ 57.9$ million saved) from the societal perspective after discounting. As a result, QIV was the dominant strategy from a societal perspective (i.e. providing more benefits and rendering cost savings), and QIV resulted in a cost per QALY gained of $€ 14,461$ (discounted) compared with TIV from a healthcare payer perspective (Table 2).

\subsection{Scenario Analysis Results}

\subsubsection{Discounting}

Varying the discount rate had a minor impact on the incremental cost-utility ratio (ICUR) (Table 2). Notably, the base case ICUR of $€ 14,461$ was based on a $3.0 \%$ discount on costs and a $1.5 \%$ discount on QALYs. With no discounting, the ICUR increased to $€ 15,375$, and with a $3.0 \%$ discount on both costs and QALYs, the ICUR increased to $€ 17,486$ from the payer perspective. From the societal perspective, QIV remained the dominant strategy versus TIV.

\subsubsection{Vaccine Price}

The price per dose of QIV was $€ 3.87$ higher than TIV in the base case, based on the German Lauer-Taxe [26]. From the payer perspective, the ICUR of QIV versus TIV was below $€ 50,000$ per QALY gained when the price difference between a dose of QIV and TIV was $€ 11.20$ or less. QIV remained cost-saving from the societal perspective for a price difference of up to $€ 6.80$ per dose. 
Table 1 Mean costs (expressed in 2014 Euro levels) and outcomes (means of 1000 simulations) for TIV vs. QIV in one influenza season, undiscounted and extrapolated to the entire German population

\begin{tabular}{|c|c|c|c|c|c|}
\hline Annual results & TIV & (Per 100,000) & QIV & $($ Per 100,000) & Incremental \\
\hline Vaccinated individuals & $25,216,739$ & $(31,020)$ & $25,216,739$ & $(31,020)$ & 0 \\
\hline Clinical influenza cases & $6,884,896$ & $(8469)$ & $6,608,390$ & $(8129)$ & $-276,506$ \\
\hline Healthy & $5,722,600$ & $(7040)$ & $5,506,204$ & $(6773)$ & $-216,396$ \\
\hline At-risk ${ }^{a}$ & $1,162,296$ & (1430) & $1,102,185$ & (1356) & $-60,111$ \\
\hline Influenza-related deaths & 4095 & (5) & 3833 & (5) & -262 \\
\hline Healthy & 2069 & (3) & 1944 & (2) & -126 \\
\hline At-risk $^{\mathrm{a}}$ & 2026 & (2) & 1890 & (2) & -136 \\
\hline Medical visits (all influenza cases excl. complications) & $2,070,517$ & $(2547)$ & $1,986,786$ & $(2444)$ & $-83,731$ \\
\hline Medical visits (complications) & 712,121 & $(876)$ & 683,669 & $(841)$ & $-28,452$ \\
\hline Hospitalisations (complications) & 99,990 & $(123)$ & 94,299 & $(116)$ & -5690 \\
\hline \multicolumn{6}{|l|}{ Payer costs breakdown } \\
\hline Cost of vaccination & $€ 576,942,623$ & $(€ 709,713)$ & $€ 674,531,402$ & $(€ 829,759)$ & $€ 97,588,779$ \\
\hline Child sickness benefit & $€ 99,150,934$ & $(€ 121,968)$ & $€ 95,770,313$ & $(€ 117,810)$ & $-€ 3,380,621$ \\
\hline Reimbursed medical costs & $€ 364,699,801$ & $(€ 448,627)$ & $€ 346,111,953$ & $(€ 425,762)$ & $-€ 18,587,848$ \\
\hline \multicolumn{6}{|l|}{ Societal costs breakdown } \\
\hline Additional vaccination costs (transportation costs) & $€ 228,715,820$ & $(€ 281,350)$ & $€ 228,715,820$ & $(€ 281,350)$ & $€ 0$ \\
\hline Non reimbursed medical costs & $€ 53,457,496$ & $(€ 65,760)$ & $€ 51,194,821$ & $(€ 62,976)$ & $-€ 2,262,675$ \\
\hline Societal non-medical costs & $€ 25,477,831$ & $(€ 31,341)$ & $€ 24,444,614$ & $(€ 30,070)$ & $-€ 1,033,217$ \\
\hline Indirect $\operatorname{costs}^{\mathrm{b}}$ & $€ 3,970,028,653$ & $(€ 4,883,641)$ & $€ 3,824,588,483$ & $(€ 4,704,731)$ & $-€ 145,440,170$ \\
\hline Net payer costs & $€ 1,040,793,358$ & $(€ 1,280,308)$ & $€ 1,116,413,668$ & $(€ 1,373,331)$ & $€ 75,620,310$ \\
\hline Net societal costs & $€ 5,318,473,158$ & $(€ 6,542,399)$ & $€ 5,245,357,406$ & $(€ 6,452,457)$ & $-€ 73,115,752$ \\
\hline Total QALYs lost & 97,066 & $(119)$ & 92,148 & (113) & -4918 \\
\hline Total LYs lost & 58,007 & (71) & 54,509 & (67) & -3498 \\
\hline
\end{tabular}

$L Y s$ life-years, $Q A L Y s$ quality-adjusted life-years, $Q I V$ quadrivalent influenza vaccine, $T I V$ trivalent influenza vaccine

a The "at-risk" population includes people at risk of complications due to influenza, including people with one or more chronic conditions, people working in the healthcare or public order section (adults only), as well as people living in residential care (elderly only) [8, 33]

${ }^{\mathrm{b}}$ Indirect costs include productivity losses as a result of absenteeism and premature mortality

\subsubsection{Calculation of Lost Productivity}

The societal perspective included costs related to productivity loss in the analyses. In the base case, productivity losses were calculated according to the human capital approach and productivity losses for caregivers caring for infants were included. When productivity losses were calculated using the friction method or when the infant caregiver costs were excluded, QIV remained dominant versus TIV.

\subsection{One-Way Sensitivity Analyses}

In univariate sensitivity analyses, key parameters were varied individually to assess the impact on the ICUR (Fig. 2) from a healthcare payer perspective. The most influential parameters were the probability of death in hospitalised individuals following respiratory tract infection, the duration of natural immunity and the disutility from an influenza episode. A longer duration of natural immunity, higher cross protection, fewer symptomatic cases, or lower vaccine efficacy all resulted in a less favourable ICUR. Higher probability of death after hospitalisation for respiratory complications or an increase in disutilities associated with influenza led to a more favourable ICUR. The ICUR under all these assumptions, however, remained under $€ 50,000$ per QALY gained. Similar results were observed from the societal perspective. For all parameter values, QIV remained dominant versus TIV.

\subsection{Probabilistic Sensitivity Analysis}

Parameter uncertainty was assessed in the PSA, where all input parameter values are simultaneously randomly sampled from their associated distributions with each simulation. The cost-effectiveness plane shows that most points in the blue cluster, representing outcomes from a societal 
Table 2 Cost-effectiveness results (means of 1000 simulations) for one influenza season, extrapolated to the entire German population, using different discount rates

\begin{tabular}{|c|c|c|c|}
\hline & TIV & QIV & Incremental \\
\hline \multicolumn{4}{|c|}{ Base case results, discount rate $(3.0 \%$ costs, $1.5 \%$ QALYs $)$} \\
\hline \multicolumn{4}{|l|}{ Vaccination costs } \\
\hline Societal perspective ${ }^{a}$ & $€ 615,937,897$ & $€ 690,378,075$ & $€ 74,440,178$ \\
\hline Payer perspective ${ }^{\mathrm{b}}$ & $€ 441,474,741$ & $€ 515,914,919$ & $€ 74,440,178$ \\
\hline \multicolumn{4}{|c|}{ Direct medical and non-medical cost of clinical influenza cases } \\
\hline Societal perspective ${ }^{a}$ & $€ 417,779,534$ & $€ 398,244,190$ & $-€ 19,535,344$ \\
\hline Payer perspective ${ }^{\mathrm{b}}$ & $€ 356,643,362$ & $€ 339,682,281$ & $-€ 16,961,081$ \\
\hline \multicolumn{4}{|l|}{ Indirect cost/productivity loss } \\
\hline Societal perspective ${ }^{a}$ & $€ 3,032,379,910$ & $€ 2,919,581,753$ & $-€ 112,798,157$ \\
\hline Payer perspective ${ }^{\mathrm{b}}$ & $€ 0$ & $€ 0$ & $€ 0$ \\
\hline \multicolumn{4}{|l|}{ Total costs } \\
\hline Societal perspective ${ }^{a}$ & $€ 4,066,097,342$ & $€ 4,008,204,019$ & $-€ 57,893,323$ \\
\hline Payer perspective ${ }^{\mathrm{b}}$ & $€ 798,118,103$ & $€ 855,597,200$ & $€ 57,479,097$ \\
\hline QALYs lost & 78,740 & 74,765 & -3975 \\
\hline ICUR societal perspective ${ }^{\mathrm{a}}$ & QIV dominates TIV & & \\
\hline ICUR payer perspective ${ }^{\mathrm{b}}$ & $€ 14,461$ & & \\
\hline \multicolumn{4}{|c|}{ Discount rate $(0 \%$ costs, $0 \%$ QALYs $)$} \\
\hline QALYs lost & 97,066 & 92,148 & -4918 \\
\hline ICUR societal perspective ${ }^{a}$ & QIV dominates TIV & & \\
\hline ICUR payer perspective ${ }^{\mathrm{b}}$ & $€ 15,375$ & & \\
\hline \multicolumn{4}{|c|}{ Discount rate $(3 \%$ costs, $3 \%$ QALYs $)$} \\
\hline QALYs lost & 65,456 & 62,166 & -3287 \\
\hline ICUR societal perspective $^{\mathrm{a}}$ & QIV dominates TIV & & \\
\hline ICUR payer perspective ${ }^{\mathrm{b}}$ & $€ 17,486$ & & \\
\hline
\end{tabular}

ICUR incremental cost-utility ratio, $Q A L Y s$ quality-adjusted life-years, $Q I V$ quadrivalent influenza vaccine, $T I V$ trivalent influenza vaccine

${ }^{a}$ Societal perspective $=$ payer perspective costs + non-reimbursed medical costs + non-medical costs + indirect costs + societal costs related to vaccination

${ }^{\mathrm{b}}$ Payer perspective $=$ reimbursed medical costs + child sickness benefit (Kinderkrankengeld) costs perspective, show an increase in QALYs at a negative incremental cost (i.e. a cost saving) for QIV versus TIV (Fig. 3a). The dots in the red cluster, representing the payer perspective, also show a gain in QALYs, but at a positive incremental cost. The majority of the cluster $(>98 \%)$, however, lies below the line indicating a cost per QALY threshold of $€ 50,000$, meaning that the ICURs were below this value (Fig. 3a). In Fig. 3b, the probability of QIV being cost-effective compared with TIV was high ( $>99 \%$, payer perspective) at a willingness-to-pay threshold of $€ 50,000$ per QALY gained, and QIV was almost always cost-saving (probability $>95 \%$ ) from the societal perspective.

\section{Discussion}

A previously published review summarised different attributes used to categorise modelling approaches for the costeffectiveness analysis of vaccines, with the first key issue to address being the choice of a static versus dynamic transmission modelling approach [27]. Dynamic models are preferred over static models to achieve valid cost-effectiveness estimates for infectious disease control, especially when herd immunity effects are crucial [11, 28, 29]. Thus, this study opted for a stochastic, individual-based and dynamic simulation approach to model the transmission of influenza. Although stochastic models are generally more complex than deterministic models based on differential equations, they can offer more realistic estimates given their flexibility to model very sophisticated features (e.g. 4Flu's highly complex immunity dynamics that allow for immunity boosting, B lineage cross protection and additional immunity loss due to new drift variants, or preferential vaccination of individuals previously vaccinated). As individual-based simulations use random numbers, individual simulation results differ (very much like different years in real populations do). Hundreds or thousands of such simulations must be averaged to estimate the true effects of vaccination strategies. Due to the repetitive 


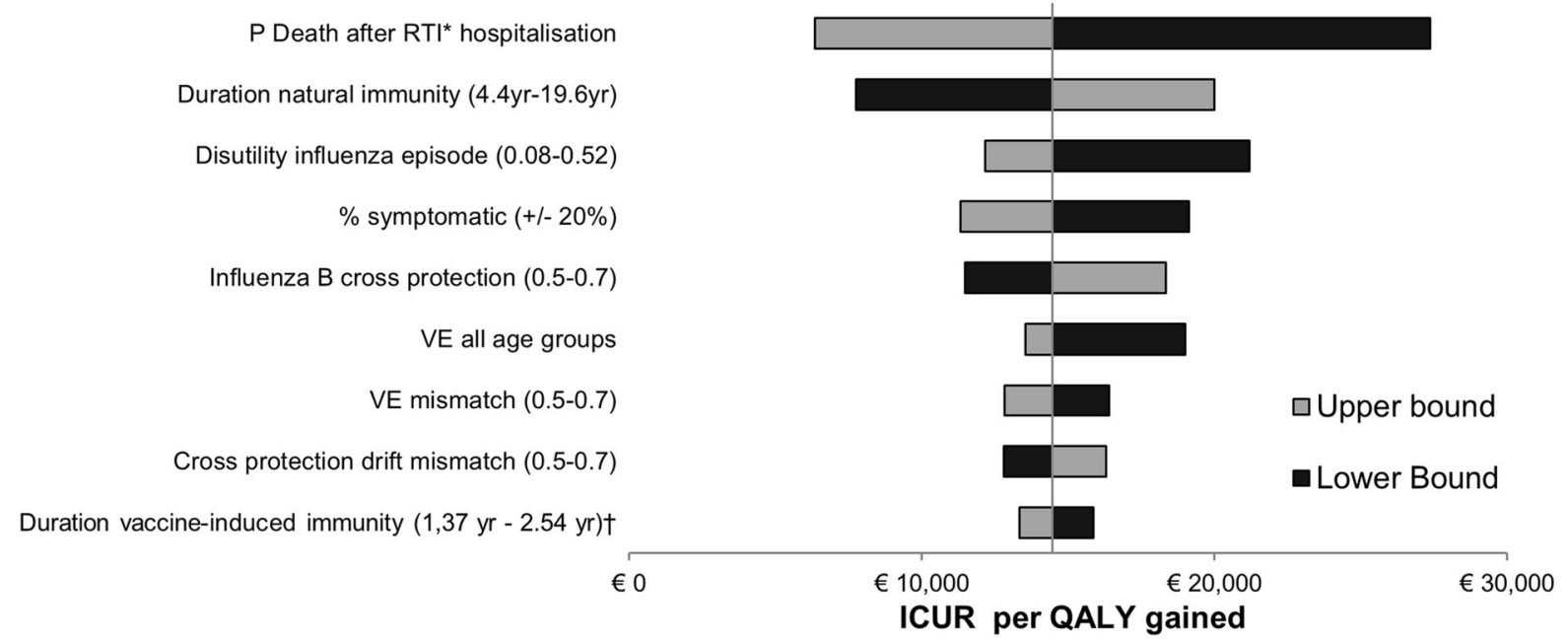

Fig. 2 Tornado diagram: one-way sensitivity analyses from the healthcare payer perspective. ICUR incremental cost-utility ratio, $P$ probability, $Q A L Y$ quality-adjusted life-year, $R T I$ respiratory tract infection, $V E$ vaccine efficacy, $y r$ year. Asterisk ranges for probability of death following hospitalisation due to respiratory tract infection are

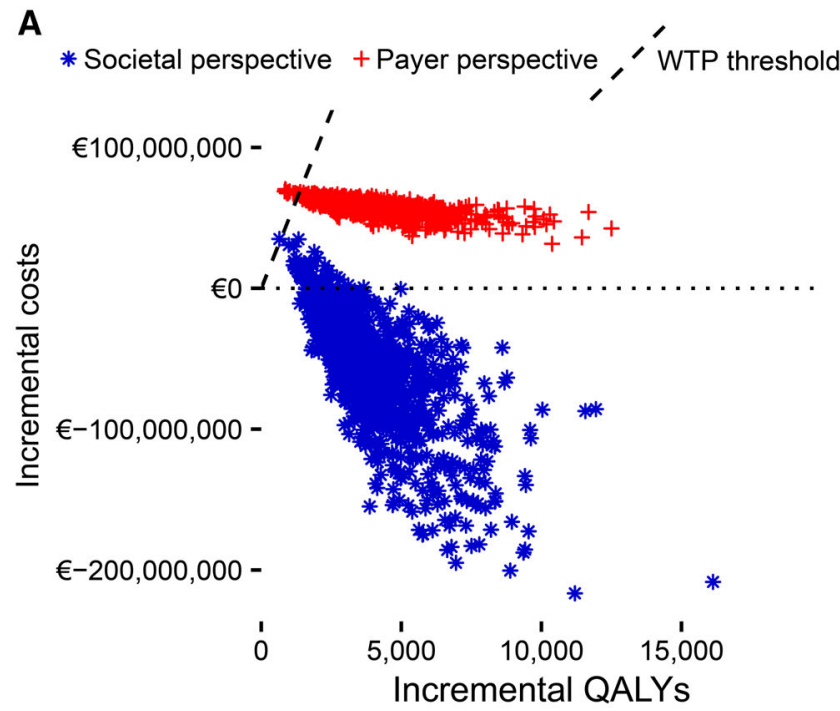

Fig. 3 Probabilistic sensitivity analysis results from societal (blue) and healthcare payer ( $\mathrm{red}$ ) perspectives: a cost-effectiveness plane for QIV vs. TIV and $\mathbf{b}$ WTP curves for QIV vs. TIV. Every one of the 1000 points in Fig. 3a represents the average result of a batch of 100 4Flu simulations, combined with one simulation that used a random

feature of this modelling approach, it was possible to introduce new drift variants in random simulation years, thus allowing the estimation of their impact on disease transmission and cost-effectiveness results. An argument against the use of more sophisticated and complex
$(0-0.01),(0-0.02)$ and $(0-0.37)$ for age groups $0-17,18-64$ and over 65 , respectively. Cross for VE, the ranges are $(0-0.82),(0-0.66)$, $(0.55-0.78),(0.55-0.69)$ and $(0.34-0.73)$ for the age groups $0-1,2-5$, $6-15,16-64$ and over 65 , respectively

B

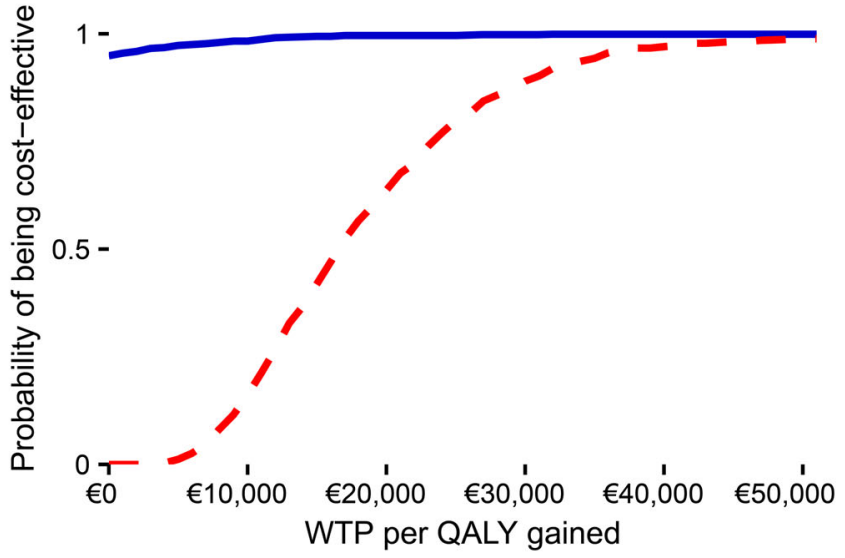

set of economic parameters. The dashed line represents an upper WTP threshold of $€ 50,000$ per QALY gained. $Q A L Y$ quality-adjusted life-year, $Q I V$ quadrivalent influenza vaccine, $T I V$ trivalent influenza vaccine, $W T P$ willingness-to-pay

simulation tools is that transparency can be lost in the process. In order to overcome this and provide maximum transparency, the 4Flu simulation model has been made publicly available on the Internet [16] and all data inputs for Germany are provided in the online resources 1 and 2 
[12]. A realistic estimate of the cost-effectiveness of QIV versus TIV was obtained by combining the 4Flu epidemiologic outcomes with economic data from a published model into the current e4Flu model.

Replacing TIV with QIV is expected to reduce the number of influenza cases and associated economic and clinical burden. The cost-effectiveness of QIV versus TIV in developed countries has been assessed using a wide range of models, from simpler decision-tree models to more complex dynamics models (e.g. compartmental dynamic transmission model or adaptive dynamic Markov chain Monte Carlo models [10, 30, 31]). Published costeffectiveness studies have shown that replacing TIV with QIV is a cost-effective strategy that reduces the burden of influenza further [8-10, 31, 32]. However, as these studies considered different perspectives (i.e. healthcare payer perspective) or did not include benefits of herd immunity (i.e. by employing static models), only this study found that replacing TIV with QIV was a dominant strategy leading to net health gains as well as net savings (from a societal perspective). Additional unique features of this study which may explain some of the differences with other studies were the inclusion of German-specific child sickness benefits ('Kinderkrankengeld') and the use of German discount rates, which may differ from other countries (i.e. higher discount rates are recommended in the UK $[14,15])$.

\subsection{Limitations}

A limitation of the current study was that international data were used to estimate input parameters for which German data were not available or lacked quality. This mainly affected estimation of the utilities and probabilities of complications. For this, international transferability was assumed to be acceptable and parameter uncertainty was taken into account in the sensitivity analysis. In addition, this study used general estimates of disease severity and progression of influenza, regardless of the influenza strain causing infection. Further research into the burden associated with specific influenza strains could improve the spatial resolution of the model. Another limitation is that the model did not include the costs and health impact from chronic conditions and rehabilitation that can result from influenza infection; therefore, the benefit of vaccination is likely to have been underestimated in this study, as in previous studies with similar omissions $[8,10]$. In addition, side effects of vaccination were not included. However, as the safety of both vaccines was assumed to be comparable, there would be no impact on the ICUR [21, 22]. Previous economic analyses of influenza vaccination have typically not included costs and QALYs linked to side effects as they are generally assumed to be mild and transient [10, 32].

\section{Conclusion}

By preventing infection from both influenza B lineages, QIV was expected to prevent more influenza cases, complications and deaths than TIV, thus providing additional health gains in Germany. As a result of reducing medical resource use and lowering productivity losses due to influenza, QIV provided more QALYs at a cost saving (dominant strategy, societal perspective) or at a cost per QALY gained of $€ 14,461$ (healthcare payer perspective). Thus, replacing TIV with QIV is likely to improve the health of the German population at a reasonable cost to the healthcare system, while providing savings to society.

Acknowledgments Authors would like to thank Business and Decision Life Sciences' platform for editorial assistance and manuscript coordination, on behalf of GSK Vaccines. Stephanie Garcia coordinated manuscript development and editorial support. The authors also thank Kavi Littlewood (Littlewood Writing Solutions, on behalf of GSK Vaccines) for providing medical writing support.

Author contributions BPN performed the literature search and provided input data for the economic model. ME and RSO conceived the mathematical model; IVV, LAVB, AA, RW, MP and CD developed the economic model; MS and ME designed the simulation tool; ME and CD ran and evaluated the simulations; all authors drafted the manuscript. All authors had full access to the data and gave final approval before submission.

\section{Compliance with Ethical Standards}

Funding GlaxoSmithKline Biologicals SA funded this study (GSK Study Identifier: HO-14-14994) and was involved in all stages of the study, including analysis of the data. GlaxoSmithKline Biologicals SA also covered all costs associated with the development and publication of this manuscript.

Conflict of interest AA, RSO and RW are employees of the GSK group of companies. RSO, AA and RW declare restricted shares ownership in the GSK group of companies. ME is a shareholder of Epimos $\mathrm{GmbH}$, which has received funding from the GSK group of companies to complete the work disclosed here as well as consultancy fees from AstraZeneca. MS is a shareholder of ExploSYS GmbH, which has received funding from Epimos $\mathrm{GmbH}$ to complete the work disclosed here. BPN's, LAVB's and IVV's companies received consultancy fees from the GSK group of companies to complete the work disclosed here as well as for other work outside this study. MP received grants and honoraria from various pharmaceutical companies, inclusive of those developing, manufacturing and marketing influenza vaccines. $\mathrm{CD}$ declares no conflict of interest.

Open Access This article is distributed under the terms of the Creative Commons Attribution-NonCommercial 4.0 International License (http://creativecommons.org/licenses/by-nc/4.0/), which 
permits any noncommercial use, distribution, and reproduction in any medium, provided you give appropriate credit to the original author(s) and the source, provide a link to the Creative Commons license, and indicate if changes were made.

\section{References}

1. WHO. Fact sheet: seasonal influenza [Internet]. 2014 [cited $2016 \mathrm{Feb}$ 4]. http://www.who.int/mediacentre/factsheets/fs211/en/. Accessed 15 Sept 2016.

2. Irving SA, Patel DC, Kieke BA, Donahue JG, Vandermause MF, Shay DK, et al. Comparison of clinical features and outcomes of medically attended influenza A and influenza B in a defined population over four seasons: 2004-2005 through 2007-2008. Influenza Other Respir Viruses. 2012;6:37-43.

3. Silvennoinen H, Huusko T, Vuorinen T, Heikkinen T. Comparative burden of influenza $\mathrm{A} / \mathrm{H} 1 \mathrm{~N} 1, \mathrm{~A} / \mathrm{H} 3 \mathrm{~N} 2$ and $\mathrm{B}$ infections in children treated as outpatients. Pediatr Infect Dis J. 2015;34: 1081-5.

4. Saisonsberichte. [cited 2016 Feb 4]. https://influenza.rki.de/ Saisonbericht.aspx. Accessed 15 Sept 2016.

5. Darvishian M, Bijlsma MJ, Hak E, van den Heuvel ER. Effectiveness of seasonal influenza vaccine in community-dwelling elderly people: a meta-analysis of test-negative design casecontrol studies. Lancet Infect Dis. 2014;14:1228-39.

6. Mitteilung der Ständigen Impfkommission am Robert Koch-Institut (RKI) Empfehlungen der Ständigen Impfkommission (STIKO) am Robert Koch-Institut/Stand: August 2015. Epidemiol Bull. 2015. http://www.rki.de/DE/Content/Infekt/ EpidBull/Archiv/2015/Ausgaben/34_15.pdf?_blob=publicationFile. Accessed 15 Sept 2016.

7. Recommended composition of influenza virus vaccines for use in the 2012-2013 northern hemisphere influenza season. [cited 2016 Feb 4]. http://www.who.int/influenza/vaccines/virus/recommendations/ 201202_recommendation.pdf?ua=1. Accessed 15 Sept 2016.

8. Van Bellinghen L-A, Meier G, Van Vlaenderen I. The potential cost-effectiveness of quadrivalent versus trivalent influenza vaccine in elderly people and clinical risk groups in the UK: a lifetime multi-cohort model. PLoS One. 2014;9:e98437.

9. Meier G, Gregg M, Poulsen Nautrup B. Cost-effectiveness analysis of quadrivalent influenza vaccination in at-risk adults and the elderly: an updated analysis in the UK. J Med Econ. 2015;18:746-61.

10. Thommes EW, Ismaila A, Chit A, Meier G, Bauch CT. Costeffectiveness evaluation of quadrivalent influenza vaccines for seasonal influenza prevention: a dynamic modeling study of Canada and the United Kingdom. BMC Infect Dis. 2015;15:465.

11. Jit M, Brisson M. Modelling the epidemiology of infectious diseases for decision analysis: a primer. Pharmacoeconomics. 2011;29:371-86

12. Eichner M, Schwehm M, Hain J, Uphoff H, Salzberger B, Knuf $\mathrm{M}$, et al. 4Flu - an individual based simulation tool to study the effects of quadrivalent vaccination on seasonal influenza in Germany. BMC Infect Dis. 2014;14:365.

13. Carrat F, Vergu E, Ferguson NM, Lemaitre M, Cauchemez S, Leach S, et al. Time lines of infection and disease in human influenza: a review of volunteer challenge studies. Am J Epidemiol. 2008;167:775-85.

14. Ultsch B, Damm $O$, Beutels $P$, Bilcke J, Brüggenjürgen $B$, Gerber-Grote A, et al. Methods for health economic evaluation of vaccines and immunization decision frameworks: a consensus framework from a European vaccine economics community. Pharmacoeconomics. 2016;34:227-44.
15. Schad M, John J. Towards a social discount rate for the economic evaluation of health technologies in Germany: an exploratory analysis. Eur J Health Econ. 2012;13:127-44.

16. Staat and Gesellschaft-Bevölkerungsstand-Bevölkerung auf Grundlage des Zensus 2011-Statistisches Bundesamt (Destatis). Internet.

17. Epimos G. 4Flu. http://www.4flu.net. Accessed 15 Sept 2016.

18. Mossong J, Hens N, Jit M, Beutels P, Auranen K, Mikolajczyk R, et al. Social contacts and mixing patterns relevant to the spread of infectious diseases. PLoS Med. 2008;5:0381-91.

19. Recommendations of the Standing Committee on Vaccination (STIKO) at the Robert Koch Institute/Effective: August 2015. Epidemiol Bull. 2015;34. http://www.rki.de/EN/Content/ infections/Vaccination/recommandations/34_2015_engl.pdf?_ blob=publicationFile. Accessed 15 Sept 2016.

20. Williams CJ, Schweiger B, Diner G, Gerlach F, Haaman F, Krause G, et al. Seasonal influenza risk in hospital healthcare workers is more strongly associated with household than occupational exposures: results from a prospective cohort study in Berlin, Germany, 2006/07. BMC Infect Dis. 2010;10:8.

21. Graaf $\mathrm{H}$ de, Faust SN. Fluarix quadrivalent vaccine for influenza. Inform Healthc. 2015. doi:10.1586/14760584.2015.1057573.

22. Bekkat-Berkani R, Ray R, Jain VK, Chandrasekaran V, Innis BL. Evidence update: GlaxoSmithKline's inactivated quadrivalent influenza vaccines. Abingdon: Taylor \& Francis; 2015. doi:10. 1586/14760584.2016.1113878.

23. Groot Koerkamp B, Weinstein MC, Stijnen T, Heijenbrok-Kal MH, Hunink MGM. Uncertainty and patient heterogeneity in medical decision models. Med Decis Making. 2010;30:194-205.

24. Krejczy M, Harenberg J, Marx S, Obermann K, Frölich L, Wehling M. Comparison of cost-effectiveness of anticoagulation with dabigatran, rivaroxaban and apixaban in patients with nonvalvular atrial fibrillation across countries. J Thromb Thrombolysis. 2014;37:507-23.

25. Brettschneider C, Luck T, Fleischer S, Roling G, Beutner K, Luppa $\mathrm{M}$, et al. Cost-utility analysis of a preventive home visit program for older adults in Germany. BMC Health Serv Res. 2015; $15: 141$

26. Lauer-Fischer-Apothekensoftware und Arzneimitteldaten. [cited 2015 Dec 1]. http://www2.lauer-fischer.de/home/. Accessed 15 Sept 2016.

27. Kim S-Y, Goldie SJ. Cost-effectiveness analyses of vaccination programmes: a focused review of modelling approaches. Pharmacoeconomics. 2008;26:191-215.

28. Brisson M, Edmunds WJ. Economic evaluation of vaccination programs: the impact of herd-immunity. Med Decis Mak. 2003;23:76-82.

29. Garnett GP. Role of herd immunity in determining the effect of vaccines against sexually transmitted disease. J Infect Dis. 2005;191(Suppl):S97-106.

30. You JHS, Ming W-K, Chan PKS. Cost-effectiveness of quadrivalent influenza vaccine in Hong Kong-a decision analysis. Hum Vaccin Immunother. 2015;11:564-71.

31. Clements KM, Meier G, McGarry LJ, Pruttivarasin N, Misurski DA. Cost-effectiveness analysis of universal influenza vaccination with quadrivalent inactivated vaccine in the United States. Hum Vaccin Immunother. 2014;10:1171-80.

32. Chit A, Roiz J, Aballea S. An assessment of the expected costeffectiveness of quadrivalent influenza vaccines in Ontario, Canada using a static model. PLoS One. 2015;10:e0133606.

33. Koch Institut R. Anhang zum Nationalen Pandemieplan (Stand: Mai 2007). 2007 [cited 2016 Jul 29]. http://www.rki.de/DE/Content/ InfAZ/I/Influenza/Pandemieplanung/Downloads/Influenzapande mieplan_Anhang.pdf;jsessionid=ADDF21773449665793E01F 883C8FE147.2_cid298?_blob=publicationFile. Accessed 15 Sept 2016. 